\title{
Entrenamiento sobre cinta rodante en niños con enfermedades respiratorias crónicas. Serie clínica
}

\author{
GREGORY VILLARROEL S.*,****, MACARENA FAÚNDEZ S.*, GONZALO MOSCOSO A.*****, \\ YORSCHUA JALIL C.* y CLAUDIA ASTUDILLO M.**,***
}

Treadmill training in children with chronic respiratory diseases: Clinical series

Introduction: Chronic respiratory diseases (CRD) in children have increased in recent years. Respiratory Rehabilitation is one of the treatments used in this population. Objective: To evaluate the impact of a treadmill training program over pediatric patients with CRD in the Josefina Martinez Children's Hospital at Santiago de Chile. Methods: Retrospective cases series with prospective record of 9 patients $7.1 \pm 3.9$ years-old with CRD and treadmill training. The patients performed 24 sessions. The Pre-post records of the 6-minute walk test $(6 \mathrm{MW})$ and the maximum speed obtained in the Incremental Load Test (ILT) were analyzed. Results: Averages of the distance traveled pre and post-training were $383 \pm 142.4$ meters and $451.7 \pm 142.4$ meters respectively $(p<0.0001)$. The average maximum speed obtained in the ILT was $4.1 \pm 1.1 \mathrm{~km} / \mathrm{h}$ and $5.4 \pm 1.27 \mathrm{~km} / \mathrm{h}(p=0.001)$. Conclusion: The distance walked in the 6-minute walk test and the maximum work capacity improve significantly with treadmill training in these patients with CRD.

Key words: Child; walk test; retrospective study; exercise; chronic respiratory disease.

\section{Resumen}

Introducción: Las enfermedades respiratorias crónicas (ERC) en niños han aumentado en los últimos años siendo la Rehabilitación Respiratoria uno de los tratamientos utilizados en esta población. Objetivo: Evaluar el impacto de un programa de entrenamiento aeróbico sobre cinta rodante en pacientes pediátricos con ERC del Hospital Josefina Martínez. Metodología: Serie retrospectiva de casos con registro prospectivo de 9 pacientes con una edad promedio de 7,1 $\pm 3,9$ años con ERC y entrenamiento aeróbico. Los pacientes realizaron 24 sesiones. Se analizaron los registros pre-post de los test de marcha de 6 min (TM6) y la velocidad máxima obtenida en el Test Cardiopulmonar Incremental (VTCI). Resultados: Las medias de la distancia recorrida en TM6 pre y post entrenamiento fueron de $383 \pm 142,4 m$ y 451,7 $\pm 142,4$ m respectivamente $(p<0,0001)$. Las medias de las VTCI pre y post entrenamiento fueron: $4,1 \pm 1,1 \mathrm{~km} / \mathrm{h}$ y $5,4 \pm 1,27 \mathrm{~km} / \mathrm{h}(p=0,001)$. Conclusiones: La distancia recorrida en el TM6 y la capacidad máxima de trabajo mejoraron significativamente con el entrenamiento aeróbico en estos pacientes con ERC.

Palabras clave: niños; Test de marcha; estudio retrospectivo; ejercicio; enfermedades respiratorias crónicas.

* Kinesiólogo, Hospital Josefina Martínez. Santiago, Chile.

** Pediatra Broncopulmonar. Hospital Josefina Martínez. Santiago, Chile.

*** Instructor Adjunto. Departamento de Enfermedades Cardiovasculares y Respiratorias del Niño. División de Pediatría. Facultad de Medicina, Pontificia Universidad Católica de Chile. Santiago, Chile.

**** Instructor Adjunto. Carrera de Kinesiología, Departamento Ciencias de la Salud, Facultad de Medicina, Pontificia Universidad Católica de Chile. Santiago, Chile. 


\section{Introducción}

En los últimos años ha existido un aumento de niños con enfermedades respiratorias crónicas (ERC), debido a factores vinculados al desarrollo tecnológico de la medicina, aplicación de nuevos tratamientos y programas de salud más efectivos ${ }^{1}$. La rehabilitación respiratoria (RR) se ha utilizado como tratamiento en esta población con el fin de disminuir los síntomas respiratorios, aumentar el rendimiento físico, la participación en las actividades de la vida diaria y mejorar la calidad de vida relacionada a la salud ${ }^{2-5}$.

La mayoría de los estudios sobre los efectos del entrenamiento aeróbico en pacientes con ERC se han realizado en adultos con enfermedad pulmonar obstructiva crónica. Sin embargo se han ido reportando experiencias en niños principalmente en asma y fibrosis quística ${ }^{6-8}$.

La efectividad del entrenamiento se puede evaluar a través de diferentes mediciones como el $\mathrm{VO}_{2}$ peak (consumo cúspide de $\mathrm{O}_{2}$ ), la función pulmonar y el test de marcha de 6 min (TM6), siendo este último una de las maneras más utilizadas debido a lo simple de realizar, su buena confiabilidad y la existencia de valores de referencia en niños ${ }^{2,9}$.

De acuerdo a estos antecedentes y a la escasa experiencia reportada en niños con ERC, el objetivo de este estudio fue evaluar el impacto de un programa de entrenamiento aeróbico sobre cinta rodante en la capacidad funcional y cardiorrespiratoria de esta población.

\section{Metodología}

Este estudio corresponde a una serie retrospectiva de casos con registro prospectivo donde se evaluó el efecto de 24 sesiones de entrenamiento aeróbico en pacientes con ERC beneficiarios del programa de RR del Hospital Josefina Martínez entre los años 2012 y 2016.

\section{Pacientes}

Participaron en el programa de RR 13 niños estables con ERC, síntomas respiratorios persistentes y actividad física limitada. Se revisaron los registros de los TM6 y test cardiopulmonar de carga incremental (TCI) previos y posteriores al entrenamiento. Se excluyeron del análisis aquellos pacientes que no completaron todas las sesiones o poseían un registro incompleto de sus TM6. Finalmente se incluyeron y analizaron 9 sujetos, 5 de ellos traqueostomizados y 4 usuarios de ventilación mecánica (VM).

\section{Protocolo de evaluación}

Previo y posterior a la ejecución del programa de entrenamiento se realizó un TM6 en un pasillo de 30 metros siguiendo las recomendaciones de la $\mathrm{ATS}^{10}$. Se registró la distancia final recorrida, la frecuencia respiratoria (FR), la frecuencia cardiaca (FC) y saturación pulsátil de oxígeno $\left(\mathrm{SpO}_{2}\right)$ utilizando oxímetro portátil $\mathrm{Nonin}^{\circledR}(\mathrm{No}$ nin advantage 9590, USA).

Se realizó en cada paciente un test cardiopulmonar de carga incremental (TCI) para niños con ERC sobre cinta rodante (Lifefitness T9i Treadmill, Illinois, USA) para establecer la intensidad máxima de trabajo ${ }^{11}$. El test se inició con un calentamiento de $3 \mathrm{~min}$, luego se incrementó la pendiente en $2 \%$ y la velocidad en $0,5 \mathrm{~km} / \mathrm{h}$ cada 1 min hasta la aparición de algún criterio de detención descrito por Torres-Castro et al. ${ }^{2}$. Se registró velocidad y pendiente máxima alcanzada durante la prueba, la $\mathrm{FC}$ y $\mathrm{SpO}_{2}$.

\section{Protocolo de entrenamiento cardiopulmonar}

El entrenamiento sobre cinta rodante consistió en 24 sesiones utilizando intervalos de intensidad moderada y alta. Las primeras 3 sesiones se utilizaron cargas de 50 y $80 \%$ de la velocidad y pendiente obtenidos en el TCI, las siguientes 6 sesiones se utilizaron intensidades del 50 y $85 \%$ y las 15 sesiones finales se trabajó con intervalos intensidad de 50 y $90 \%$ (Figura 1 ). Se realizaron

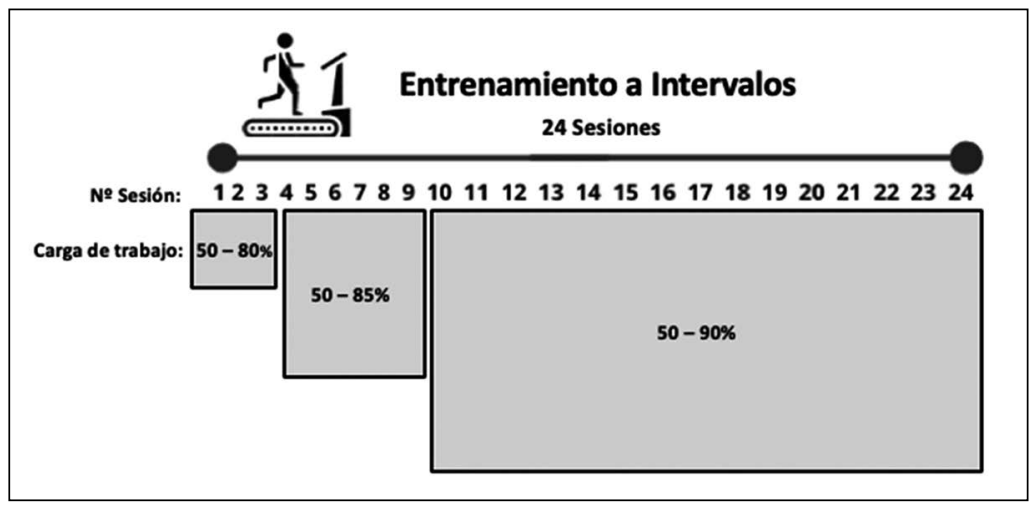

Figura 1. Esquema de cargas utilizadas en cada sesión del programa de entrenamiento. 
2-3 sesiones por semana supervisadas por kinesiólogo. Cada sesión contó con un calentamiento de $3 \mathrm{~min}, 32 \mathrm{~min}$ de trabajo a intervalos, y $3 \mathrm{~min}$ de vuelta a la calma. Siempre se mantuvo monitorización de $\mathrm{FC} \mathrm{y} \mathrm{SpO}_{2}$. En cada sesión se registró: fecha, duración de la sesión, intensidades de trabajo utilizadas y los signos vitales al inicio y final.

Las variables a analizar del TM6 fueron: edad, sexo, diagnóstico, distancia recorrida, FC, FR y $\mathrm{SpO}_{2}$ en el estado basal e inmediatamente al finalizar el TM6. Del TCI se analizaron las velocidades máximas alcanzadas.

Este estudio contó con la aprobación del comité de ética en investigación del Servicio de Salud Metropolitano Suroriente.

\section{Análisis estadístico}

Las variables de interés se describieron con medidas de frecuencia, tendencia central, porcentajes y dispersión según correspondió.

Se establecieron las diferencias para muestras pareadas por medio de la prueba sign-rank de Wilcoxon. Para las muestras independientes se utilizó t-test con varianzas homogéneas. Se consideró un nivel de significancia de $\mathrm{p}<0,05$. El análisis estadístico se realizó con STATA 13.

\section{Resultados}

Ingresaron 13 pacientes al programa de entre- namiento en cinta rodante. Cuatro no finalizaron las 24 sesiones ( 3 altas y 1 no poseía los registros completos de TM6) por lo que se incluyeron en el análisis 9 niños (Tabla 1) con una media de edad de $7,1 \pm 3,9$ años.

Tras la realización de 24 sesiones de entrenamiento, todos los pacientes mejoraron su distancia recorrida en el TM6 (Figura 2), donde el incremento promedio en metros fue de $67,9 \pm 23,7 \mathrm{~m}(17,8 \%)$ respecto a la distancia recorrida en el TM6 basal $(p=0,001)$.

Luego del entrenamiento en cinta rodante, la velocidad máxima obtenida en el TCI también incrementó significativamente $(\mathrm{p}=0,001)$. Las variables fisiológicas ( $\mathrm{FC}, \mathrm{FR}, \mathrm{SpO}_{2}$ ) evaluadas en los TM6 previo y posterior al programa de entrenamiento no mostraron diferencias estadísticamente significativas (Tabla 2).

Al comparar la distancia recorrida media del TM6 según el uso o no de ventilación mecánica (VM), pudimos observar que en los TM6 previos al programa de entrenamiento aquellos pacientes usuarios de VM caminaron menos $(297,6 \mathrm{~m})$ que los pacientes no ventilados $(491,5 \mathrm{~m})(\mathrm{p}=0,015)$. De la misma manera, las distancias recorridas luego del programa de entrenamiento fueron significativamente menores en el grupo ventilado $(364,8 \mathrm{~m})$ comparado con aquellos pacientes que no utilizaban la VM $(560,4 \mathrm{~m})(\mathrm{p}=0,014)$ (Figura 3). En esta muestra no existieron eventos adversos asociados a los TM6, TCI, ni durante las sesiones de entrenamiento.

Tabla 1. Características de los pacientes, distancia recorrida del TM6 y velocidad obtenida en el test cardiopulmonar de carga incremental

\begin{tabular}{|cccccccccc|}
\hline Sujetos & Edad & Sexo & Diagnóstico & TQT & VM & $\begin{array}{c}\text { TM6 pre } \\
(\mathbf{m})\end{array}$ & $\mathbf{\%} \mathbf{\Delta}$ TM6 & $\begin{array}{c}\text { TCI pre } \\
(\mathbf{k} / \mathbf{h})\end{array}$ & $\% \mathbf{T}$ TCI \\
\hline 1 & 5 & M & BOPI & SI & Continua & 261 & 21,5 & 4,6 & 28,3 \\
\hline 2 & 3 & M & DBP & SI & Continua & 273 & 26,4 & 1,5 & 100,0 \\
\hline 3 & 4 & F & DBP & SI & Continua & 248 & 24,2 & 3,5 & 25,7 \\
\hline 4 & 9 & M & DBP & SI & Nocturna & 420 & 21,4 & 4,5 & 24,4 \\
\hline 5 & 3 & F & OVAS & NO & Nocturna & 286 & 20,3 & 4,8 & 14,6 \\
\hline 6 & 11 & F & DCP & NO & NO & 566 & 4,2 & 5,4 & 29,6 \\
\hline 7 & 5 & F & BOPI & NO & NO & 316 & 23,4 & 4,4 & 13,6 \\
\hline 8 & 10 & M & DCP & NO & NO & 448 & 24,3 & 4,4 & 22,7 \\
\hline 9 & 14 & M & BOPI & NO & NO & 636 & 10,8 & 4,3 & 55,8 \\
\hline
\end{tabular}

BOPI: Bronquiolitis obliterante post infecciosa. DBP: Displasia broncopulmonar. DCP: Diskinesia ciliar primaria. ENM: enfermedad neuromuscular, OVAS: obstrucción de vía aérea superior. TQT: traqueostomía. VM: ventilación mecánica. \% $\Delta$ TM6: porcentaje de cambio de la distancia recorrida entre test de marcha 6 min pre y post entrenamiento. $\% \Delta$ TCI: porcentaje de cambio de la velocidad máxima del test cardiopulmonar incremental pre y post entrenamiento. 


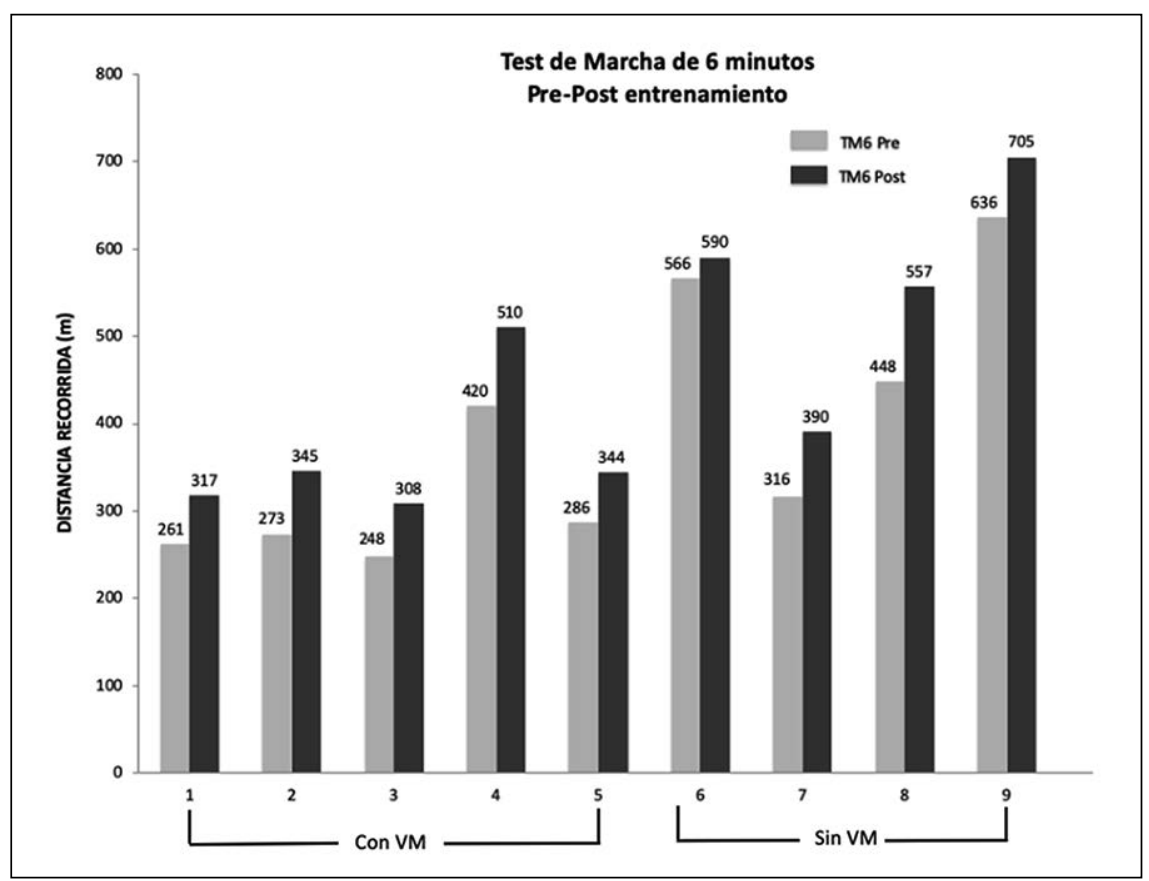

Figura 2. Distancia recorrida en los test de marcha 6 min (TM6) pre y post entrenamiento para cada participante según necesidad de ventilación mecánica.

Tabla 2. Valores obtenidos en las variables fisiológicas del TM6 y TCI

\begin{tabular}{|lccc|}
\hline Variables de los TM6 & Pre & Post & p value \\
\hline FC basal $(\mathrm{lpm})$ & $106 \pm 19,3$ & $98,7 \pm 12$ & 0,13 \\
\hline FC final $(\mathrm{lpm})$ & $125 \pm 25,6$ & $128 \pm 18,1$ & 0,72 \\
\hline FR basal $(\mathrm{rpm})$ & $30,7 \pm 9$ & $27,77 \pm 11$ & 0,63 \\
\hline FR final $(\mathrm{rpm})$ & $36,9 \pm 11$ & $33,8 \pm 11$ & 0,44 \\
\hline $\mathrm{SatO}_{2}$ basal $(\%)$ & $95,4 \pm 3$ & $95,3 \pm 2,3$ & 0,89 \\
\hline $\mathrm{SatO}_{2}$ final $(\%)$ & $93,5 \pm 3,8$ & $93,7 \pm 2.9$ & 0,88 \\
\hline Distancia recorrida en $\mathrm{TM}, \mathrm{m}$ & $383 \pm 142,4$ & $451,7 \pm 142,4$ & $0,001^{*}$ \\
\hline Velocidad máxima $\mathrm{TCI}, \mathrm{km} / \mathrm{h}$ & $4,1 \pm 1,1$ & $5,4 \pm 1,27$ & $0,001^{*}$ \\
\hline
\end{tabular}

TM6: Test de Marcha 6 min, TCI: Test Cardiopulmonar de carga Incremental. lpm: latidos por minuto, rpm: respiraciones por minuto. Cada cifra presenta la media \pm su desviación estándar. *Diferencia estadísticamente significativa.

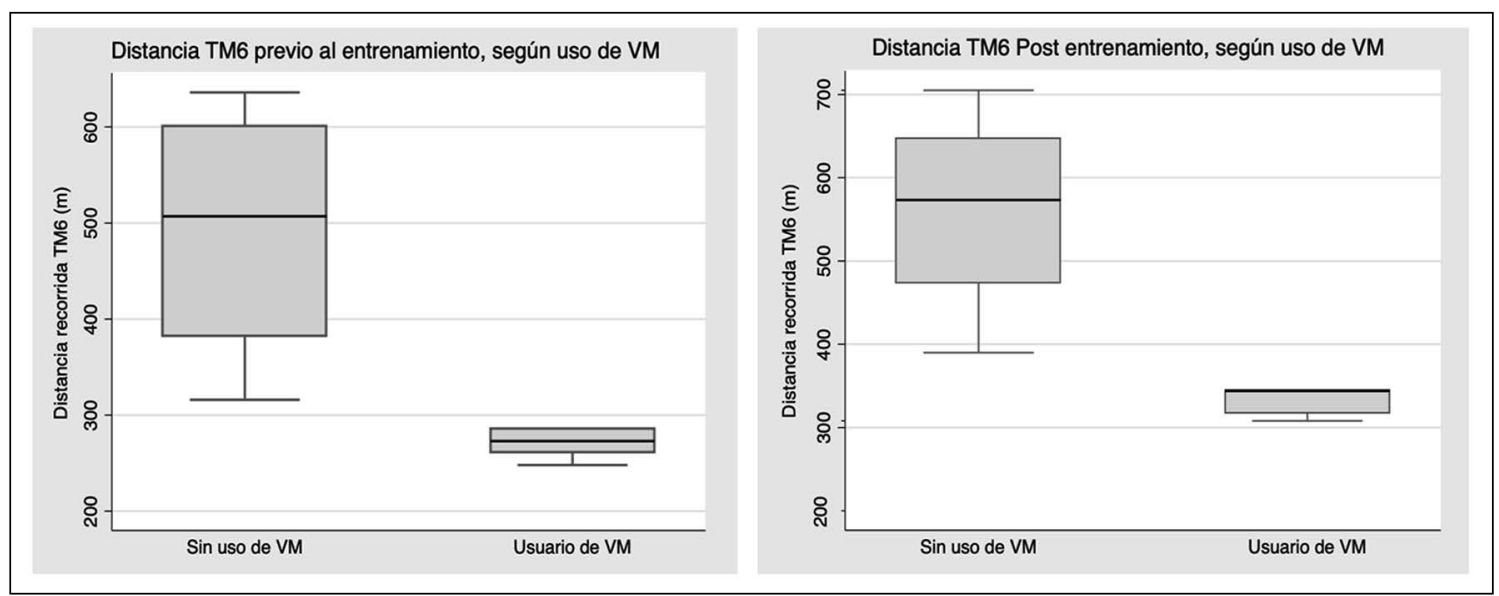

Figura 3. Distancia Recorrida en los Test de Marcha 6 min pre y post entrenamiento según uso de ventilación mecánica (VM). 


\section{Discusión}

El TM6 y el TCI permitieron valorar la capacidad funcional y cardiorrespiratoria luego de un protocolo de entrenamiento aeróbico en niños con ERC. En esta muestra la distancia recorrida en el TM6 mejora significativamente luego del entrenamiento aeróbico. Este incremento fue reportado anteriormente por Gruber et al. y Latorre-Román et al, quienes observaron mejorías significativas del 4\% y $23,9 \%$, respectivamente en el TM6 luego de un protocolo de entrenamiento aeróbico en niños con fibrosis quística y asma ${ }^{12,13}$.

En relación a las variables fisiológicas evaluadas en el TM6, Moalla et al., observaron un comportamiento similar al de este estudio en relación a la FC en niños con enfermedad cardiaca congestiva, atribuyendo esta respuesta a cambios en el tono autonómico como resultado de un entrenamiento de resistencia ${ }^{14}$.

El incremento promedio de 67 metros observado en este trabajo luego de un programa de entrenamiento podría ser clínicamente relevante para los niños con ERC. En pediatría no existen muchos reportes al respecto. McDonald et al. estimaron que la mínima diferencia clínicamente importante (MDCI) en niños con Distrofia muscular de Duchenne es de 28,5 metros ${ }^{15}$. Se hace necesario poder determinar estas diferencias clínicamente significativas en la población pediátrica con ERC.

En el presente estudio se pudo observar que los pacientes con necesidad de ventilación mecánica presentaban una menor capacidad funcional representada por la distancia recorrida en el TM6 inicial, sin embargo, pese a esta menor capacidad pudieron participar en un programa de entrenamiento sobre cinta rodante sin presentar eventos adversos y con un incremento significativo en la distancia recorrida luego del protocolo de entrenamiento.

Dentro de las limitaciones de este estudio se encuentra su carácter retrospectivo, el bajo número de sujetos y la ausencia de grupo control, siendo necesarios nuevos estudios con diseños y número de pacientes que permitan establecer causalidad entre el entrenamiento y los beneficios aquí descritos, así como también los mecanismos fisiológicos involucrados en la mejoría de la capacidad física de los niños con enfermedades respiratorias crónicas.

\section{Conclusiones}

Se observó un aumento estadísticamente significativo en la distancia recorrida del TM6 y la velocidad máxima obtenida en el TCI en los niños con ERC tras un programa de entrenamiento aeróbico. Estas mejoras se lograron en todos los pacientes que fueron entrenados independiente de su diagnóstico de base, del uso o no de traqueostomía y del tipo de asistencia ventilatoria que poseía.

De acuerdo a este trabajo es posible incorporar de manera segura a un programa de entrenamiento aeróbico a aquellos pacientes pediátricos con ERC que cumplan con los adecuados criterios de selección, incluso si presentan una menor capacidad funcional y mayor dependencia tecnológica como ocurrió con los pacientes usuarios de ventilación mecánica.

\section{Bibliografía}

1.- ZENTENO D, PUPPO H, VERA R, TORRESCASTRO R, CHUNG-YANG K, SALINAS P, et al. Guías de rehabilitación para niños con enfermedades respiratorias crónicas. Neumol Pediatr 2008; 3: 25-31.

2.- TORRES-CASTRO R, ZENTENO D, RODRÍGUEZ NI, VILLARROEL G, ÁLVAREZ C, GATICA D, et al. Guías de Rehabilitación Respiratoria en Niños con Enfermedades Respiratorias Crónicas : Actualización 2016. Pulmonary Rehabilitation Guidelines in Children with Chronic Respiratory. Neumol Pediatr 2016; 11: 114-31.

3.- JALIL Y, VILLARROEL G, MOSCOSO G, BARAÑAO P, MENDEZ M. Role of respiratory physiotherapists in the discharge process in children with long-term mechanical ventilation. Neumol Pediatr 2017; 12: 1618.

4.- SPRUIT MA, SINGH SJ, GARVEY C, ZUWALLACK R, NICI L, ROCHESTER C, et al. An official American Thoracic Society/European Respiratory Society statement: key concepts and advances in pulmonary rehabilitation. Am J Respir Crit Care Med [Internet] 2013; 188: e13-64. Disponible en: http:// www.ncbi.nlm.nih.gov/pubmed/24127811.

5.- BASARAN S, GULER-UYSAL F, ERGEN N, SEYDAOGLU G, BINGOL-KARAKOÇ G, UFUK ALTINTAS D. Effects of physical exercise on quality of life, exercise capacity and pulmonary function in children with asthma. J Rehabil Med 2006; 38: 130-5.

6.- BRADLEY J, MORAN F. Physical training for cystic fibrosis (Review). Cochrane Database Syst Rev 2011; (5).

7.- FRANCOIS-PIERRE COUNIL, ALAIN VARRAY SM. Training of aerobic and anaerobic fitness in children with asthma. J Pediatr 2003; 142: 179-84.

8.- RAM FSF, ROBINSON S, BLACK PN PJ. Physical training for asthma (Review). Cochrane Database Syst Rev 2009; 16 (5). 
9.- GATICA D, PUPPO H, VILLARROEL G, MARTÍN IS, LAGOS R, MONTECINO JJ, et al. Reference values for the 6-minutes walking test in healthy Chilean children | Valores de referencia del test de marcha de seis minutos en niños sanos. Rev Med Chile 2012; 140: 1014-21.

10.- CRAPO RO, CASABURI R, COATES AL, ENRIGHT PL, MACINTYRE NR, MCKAY RT, et al. ATS statement: Guidelines for the six-minute walk test. Am J Respir Crit Care Med 2002; 166: 111-7.

11.- VEGA-BRICEÑO LE, ZENTENO D, ABARA S, ALVAREZ C, ARANCIBIA JC, ARCE JD, et al. Guía clínica para el diagnóstico y cuidado deniños/adolescentes con bronquiolitis obliterantepost-infecciosa, 2009. Neumol Pediatr 2009; 25: 141-63. Disponible en: http:// www.neumologiapediatrica.cl/pdf/20094Suplemento/ GuiaClinica.pdf\%5Cnpapers2://publication/uuid/ E385C9EC-8E97-43A0-9708-F3F5D85C24A9.

12.- GRUBER W, ORENSTEIN DM, BRAUMANN KM, HU G. Health-Related Fitness and Trainability in Chil- dren With Cystic Fibrosis.Pediatric Pulmonology 2008; 964: 953-64.

13.- LATORRE-ROMÁN PÁ, NAVARRO-MARTÍNEZ AV, GARCÍA-PINILLOS F. The effectiveness of an indoor intermittent training program for improving lung function, physical capacity, body composition and quality of life in children with asthma. J Asthma 2014; 51: 544-51.

14.- MOALLA W, GAUTHIER R, MAINGOURD Y, AHMAIDI S. Six-minute walking test to assess exercise tolerance and cardiorespiratory responses during training program in children with congenital heart disease. Int J Sports Med 2005; 26: 756-62.

15.- MCDONALD CM, HENRICSON EK, ABRESCH RT, FLORENCE J, EAGLE M, GAPPMAIER E, et al. The 6-minute walk test and other clinical endpoints in Duchenne muscular dystrophy: Reliability, concurrent validity, and minimal clinically important differences from a multicenter study. Muscle and Nerve 2013; 48: 357-68.

Correspondencia a:

Klgo. Gregory Villarroel S.

Hospital Josefina Martínez

Camilo Henríquez 3691,

Santiago de Chile

Email: grvillarroel@uc.cl 